\title{
The Advance in Self-healing Scheme of Polymeric Materials
}

\section{Tao Xu*}

School of Civil Engineering, Nanjing Forestry University, Nanjing 210037, China

Polymeric materials are increasingly used in a variety of engineering fields, such as autos, ships, aircrafts, bridges, buildings, etc. due to their light weight, high specific strength and corrosion resistance. However, the impact or ageing is a common problem that induces considerable damages in these polymer based materials, compromising their structural integrity and service life. To heal the damages in the polymeric materials, some self-healing schemes are developed to restore their mechanical properties and extend the service life. The existing main self-healing schemes include the micro-capsule approach, the micro-channel approach, the thermoplastic additive approach, the intrinsic approach, etc.

For the first scheme, a liquid healing agent is encased in microcapsules. When an approaching crack ruptures the capsule dispersed in the polymeric materials with catalysts throughout the matrix, the healing agent is released to fill in the crack. Catalyst in the crack vicinity initiates an in-situ polymerization reaction such as ring-opening metathesis polymerization and thus patches the crack, achieving the self-healing purpose. Similarly, in the micro-channel method, the liquid healing agent is enclosed in hollow fibers which are dispersed in materials to mimic the vascular networks of humans or animals. When the crack fractures the fibers, the healing agent is released to fill in the crack and heal the damage. For the thermoplastic additive approach, the healing is achieved through the melting and physical bonding of the incorporated thermoplastic to the matrix. In the intrinsic approach, the self-healing is achieved through thermally reversible reactions of the matrix, ionomic coupling, molecular diffusion and even hydrogen bonding. Although the above schemes are very effective in self-healing micro-length scale damages, a tremendous challenge which they face is how to heal macroscopic or structural scale damages autonomously, repeatedly, efficiently, and at molecular length scale.

This is because structural scale damages need a sufficient amount of healing agent to fill in the crack before the fractured surfaces can be bonded. However, the incorporation of a large amount of healing agent may significantly alter the physical and mechanical properties of the host structure, affecting adversely the strength, structural load bearing and dimensional stability of the matrix. Further, the hollow residues of capsules or hollow fibers themselves may become potential defects when the encased healing agent outflows. Although the ionomer has been proved to self-heal ballistic impact damage, it inherently utilized the rebound of the broken ionomer pieces and the heat created by projectile perforation. Without the elastic rebound and the heat, the broken pieces cannot be brought into contact and ionomer molecules cannot self-heal themselves, either. Another method that has been demonstrated to close cracks in polymeric materials involved the use of shape memory alloy wires with dispersed microcapsules, but the use of microcapsules may also limit the healing repeatability. Hence, innovative ways must be developed in order to achieve a reasonable healing efficiency with minimal sacrifice of structural capacity.

To overcome the above problems of exiting self-healing approaches, a sequential two-step healing scheme was proposed by mimicking the biological healing process of wound healing in humans: Close Then Heal (CTH). In this scheme, the confined shape recovery of shape memory polymers were utilized as the first step to close and seal macroscopic cracks or structural scale damages before the existing self-healing mechanisms such as microcapsule, hollow fibers or thermoplastic particles take effect. This is achieved through activating the programmed or educated shape memory polymer to recover its original shape under external confined conditions upon heated above its glass transition temperature. This confinement helps in fully closing the crack.

Then the microscopic or molecular-scale healing was achieved by melting the thermoplastic particles dispersed in the matrix. Since the cracks were closed or sealed first, less thermoplastic particles were required to heal the damages. This scheme has been validated using crack-sensitive three-point bending tests of polymeric materials by dispersing copolyester particles in a polystyrene shape memory polymer matrix. It is believed that the $\mathrm{CTH}$ scheme can ensure a similar healing efficiency with a lesser sacrifice in host material properties as compared with other exiting seal-healing schemes.

In practical applications, the external confinement can be achieved by architectural design of engineering structures. This CTH scheme lays a solid foundation for self-repairing structural scale damages of shape memory polymer based materials. It is envisioned that if the existing micro-length scale healing scheme such as microcapsules or thermoplastic particles are incorporated into the shape memory polymer matrix, the $\mathrm{CTH}$ scheme could be achieved repeatedly, efficiently, almost autonomously, and at molecular-length scale, satisfying all the self-healing criteria. Also the CTH basic idea would be further applied in other fields, for example, civil engineering, mechanical engineering, etc.
*Corresponding author: Tao Xu, School of Civil Engineering, Nanjing Forestry University, Nanjing 210037, China, E-mail: seuxt@hotmail.com

Received July 26, 2012; Accepted July 28, 2012; Published July 31, 2012

Citation: Xu T (2012) The Advance in Self-healing Scheme of Polymeric Materials J Material Sci Eng 1:e101. doi:10.4172/2169-0022.1000e101

Copyright: ( $) 2012 \mathrm{Xu} \mathrm{T}$. This is an open-access article distributed under the terms of the Creative Commons Attribution License, which permits unrestricted use, distribution, and reproduction in any medium, provided the original author and source are credited. 